\title{
OPTIMAL CONTROL OF A TWO-STAGE HYBRID MANUFACTURING SYSTEM MODEL*
}

\author{
Christos G. Cassandras, Qinjia Liu and Kagan Gokbayrak \\ Department of Manufacturing Engineering \\ Boston University \\ Boston, MA 02215 \\ cgc@bu . edu , qjliu@bu . edu , kgokbayr@bu . edu \\ David L. Pepyne \\ Division of Engineering and Applied Sciences \\ Harvard University \\ Cambridge, MA 02138 \\ pepyne@hrl.harvard.edu
}

\begin{abstract}
Extending previous work for an optimal control problem of a single-stage system, we consider a two-stage manufacturing system where each job has a physical state characterized by time-driven dynamics and a temporal state by event-driven dynamics. We derive necessary conditions for optimality and develop some new algorithms for explicit solution of the problem that make use of Bezier approximation techniques. In addition, we establish some properties of the optimal control sequence that have interesting implications.
\end{abstract}

\section{Introduction}

The term "hybrid" is used to characterize systems that combine time-driven and event-driven dynamics. The former are represented by differential (or difference) equations, while the latter may be described through various frameworks used for Discrete Event Systems (DES), such as timed automata, max-plus equations, queueing networks, or Petri nets (see [4]). Broadly speaking, two categories of modeling frameworks have been proposed to study hybrid systems: Those that extend event-driven models to include time-driven dynamics; and those that extend the traditional timedriven models to include event-driven dynamics; for an overview, see [11],[1],[2],[3].

*This work was supported in part by the National Science Foundation under Grants EEC-95-27422 and ACI-98-73339, by AFOSR under contract F49620-98-1-0387, by the Air Force Research Laboratory under contract F30602-97-C-0125, and by EPRI under contract WO8333-03.
The hybrid system modeling framework used in this paper falls into the first category above and is motivated by the structure of many manufacturing systems. In these systems, discrete entities (referred to as jobs) move through a network of workcenters which process the jobs so as to change their physical characteristics according to certain specifications. Associated with each entity is a two-component state-vector consisting of a temporal component and a physical component. The temporal state component tracks event occurrence time information such as the arrival time, waiting time, service time, or departure time of the job at the various workcenters and evolves according to "max-plus" recursive equations. The physical state component evolves according to time-driven dynamics modeled through differential equations which, depending on the particular problem being studied, describe changes in such quantities as the temperature, size, weight, chemical composition, bacteria level, or some other measure of the "quality" of the job. The temporal and physical components interact in that the initial physical state and time period over which the physical state is allowed to evolve are determined by the occurrence times of certain controllable events. The interaction of time-driven with event-driven dynamics leads to a natural trade-off between temporal requirements on job completion times and physical requirements on the quality of the completed jobs. For example, while the physical state of a job can be made arbitrarily close to a desired "quality target," this usually comes at the expense of long processing times resulting in excessive inventory costs or violation of constraints on job completion deadlines. Our objective, therefore, is to formulate and solve optimal control problems associated with such trade-offs. 
In earlier work [5],[9],[7],[6],[12] we used this framework to analyze a single-stage manufacturing process assuming a deterministic setting, i.e., a known job arrival schedule and controllable processing times for all jobs. In this paper, under the same setting, we extend our previous results to a network consisting of two servers in tandem. While this may at first seem like a simple extension, it turns out to be a much more difficult problem than the single-server case as far as obtaining explicit solutions is concerned.

\section{Review of Single-Stage Case}

In [12] we studied the following hybrid system optimal control problem,

$$
\min _{u_{1}, \ldots, u_{N}} J=\sum_{i=1}^{N}\left(\alpha u_{i}^{2}+\beta x_{i}^{2}\right)
$$

subject to

$$
x_{i}=\max \left(x_{i-1}, a_{i}\right)+\frac{q}{u_{i}}
$$

for a given sequence $0=a_{0} \leq a_{1} \leq a_{2} \leq \ldots \leq a_{N}<$ $\infty$. Equations (1) and (2) describe a single-server manufacturing system. We have $N$ discrete jobs, indexed by $i=1,2, \ldots, N$. Job $i$ arrives for processing at some given time $a_{i}$. The job waits in a queue until its turn comes to be processed. Jobs are processed one at a time on a First-Come, First-Served (FCFS), nonpreemptive basis. The physical dynamics of each job, $z_{i}$, are assumed here to have the form

$$
\dot{z}_{i}=u_{i}
$$

with $z_{i}(0)=0$, and each job is processed until its final state reaches $q$. The control determines the service time required to reach this desired final state. The job departs the server at time $x_{i}$. The cost trades off large controls (which correspond to short service times) against large departure times (which may be related to missed deadlines associated with jobs).

The existence of a unique optimal solution for the above problem was established in [8]. To develop numerical algorithms to solve the problem we used standard calculus of variations techniques and took advantage of the decomposition of an optimal state trajectory into decoupled segments corresponding to the "busy periods" of the underlying queueing system. A crucial feature of the problem manifests itself when one considers the necessary conditions for optimality, which in this case consist of the optimality equation,

$$
0=2 \alpha u_{i}-\lambda_{i} \frac{q}{u_{i}^{2}}
$$

where $\lambda_{i}$ is a costate variable, $i=1, \ldots, N$; the costate equation,

$$
\lambda_{i}=2 \beta x_{i}+\lambda_{i+1} \frac{d \max \left(a_{i+1}, x_{i}\right)}{d x_{i}}, \lambda_{N}=2 \beta x_{N}
$$

and the state equation (2). Note that in the costate equation, $\max \left(a_{i+1}, x_{i}\right)$ is not differentiable when $x_{i}=$ $a_{i+1}$. We define the $i$ th job to be critical if the optimal solution is such that $x_{i}=a_{i+1}$. It turns out [7],[6] that critical jobs are a common occurrence in an optimal trajectory. For some cost functions, in fact, every job being critical constitutes an optimal solution (intuitively corresponding to a "just-in-time" control policy). When critical jobs are part of an optimal solution, however, a standard gradient-based procedure for solving the Two-Point Boundary-Value Problem (TPBVP) in (5)-(2) will not work. For this reason, identification of the conditions leading to critical jobs is an essential component of the analysis and solution of such problems.

One such necessary and sufficient condition was presented in [6]. Formally, one can use results from nonsmooth optimization theory (see [10]) that apply to locally Lipschitz continuous functions, such as the cost function for our problem. In particular, the subdifferential of a locally Lipschitz continuous function $f(u)$ at $u \in R^{N}$ is denoted by $\partial f(u)$ and has the following properties: $(i) \partial f(u)$ is a nonempty, compact and convex set in $R^{N}$, (ii) $\partial f(u)$ is a singleton iff $f$ is continuously differentiable in some open set containing $u$, in which case $\partial f(u)=\{\nabla f(u)\}$, (iii) If $u$ is a local minimum of $f$, then $0 \in \partial f(u)$. While in general, the subdifferential is a set in $R^{N}$, for the single-server system it is easily obtained as the union of a set of intervals.

Before we define these intervals, let us recall definitions of busy and idle periods as periods of time during which the server is busy serving jobs and not serving jobs respectively. Busy periods can further be partitioned into blocks which start with the first job after either a critical job or an idle period and end with either a critical job or the last job of the busy period. For any job $i=1, \ldots, N$ let $n(i)$ be the index of the last job in the busy period that contains job $i$, and $m(i)$ be the index of the last job in the block that contains job $i$. Thus, define

$$
\begin{aligned}
n(i) & =\min \left\{n \geq i: x_{n}<a_{n+1}\right\} \\
m(i) & =\min \left\{m \geq i: x_{m} \leq a_{m+1}\right\}
\end{aligned}
$$

Then the subdifferential associated with job $i$ is defined by the interval $\left[\xi_{i}^{-}, \xi_{i}^{+}\right]$where

$$
\begin{aligned}
& \xi_{i}^{-}=2 \alpha u_{i}-\frac{q}{u_{i}^{2}} \sum_{j=i}^{m(i)} 2 \beta x_{j} \\
& \xi_{i}^{+}=2 \alpha u_{i}-\frac{q}{u_{i}^{2}} \sum_{j=i}^{n(i)} 2 \beta x_{j}
\end{aligned}
$$


and the condition for optimality requires,

$$
0 \in\left[\xi_{i}^{-}, \xi_{i}^{+}\right]
$$

for each $i=1, \ldots, N$. Using this definition, we were able to identify properties of $\xi_{i}^{-}, \xi_{i}^{+}$that were used to develop several efficient numerical algorithms for solving the problems involving single-server systems $[7],[6],[8]$.

\section{The Two-Stage Case}

In this paper we study a system consisting of a tandem connection of two single-server queues. It turns out that this is a much more difficult problem than the single-server case. To explain, we consider the following optimal control problem,

$\min _{u_{1,1}, \ldots, u_{N, 1}, u_{1,2}, \ldots, u_{N, 2}} J=\sum_{i=1}^{N}\left\{\alpha_{1} u_{i, 1}^{2}+\alpha_{2} u_{i, 2}^{2}+\beta x_{i, 2}^{2}\right\}$

subject to

$$
\begin{aligned}
& x_{i, 1}=\max \left(a_{i}, x_{i-1,1}\right)+\frac{q_{1}}{u_{i, 1}} \\
& x_{i, 2}=\max \left(x_{i, 1}, x_{i-1,2}\right)+\frac{q_{2}}{u_{i, 2}}
\end{aligned}
$$

Similar to the single-server example, the objective is to process $N$ jobs. Job $i$ arrives to the first server at a given time $a_{i}$. The job waits in a FCFS queue for processing by the first server. The job is processed in the first server until its physical state reaches a value $q_{1}$. The job departs the first server and enters a FCFS queue at a second server at time $x_{i, 1}$. The job is processed in the second server until its physical state reaches $q_{2}$. The job then departs the system at time $x_{i, 2}$. The optimal control objective is to determine $u_{i, 1}$ and $u_{i, 2}$, the controls at server 1 and 2 respectively, for each job $i=1, \ldots, N$, to keep the completion time small, while at the same time ensuring that the service time is not too short.

While the above problem might appear to be a simple extension of the single-server case, it turns out to be much more difficult. As in the single-server case, the cost is a Lipschitz continuous function, and the solution reduces to finding the minimum of this function. As discussed previously, this involves finding a solution for which the zero vector is a member of the subdifferential. Whereas, in the single-server case, the subdifferential was composed of a union of easily determined intervals, for even this simple two-server case, it is not clear how to obtain the subdifferential. Without the subdifferential, it is not possible even to check candidate solutions, and, hence, it is difficult to develop numerical algorithms for solving the problem. Our approach for obtaining a solution for the tandem case, therefore, will not employ the subdifferential as it did in the single-server case, as explained below.

We begin by forming the augmented cost,

$$
\begin{aligned}
\bar{J}= & \sum_{i=1}^{N}\left\{\alpha_{1} u_{i, 1}^{2}+\alpha_{2} u_{i, 2}^{2}+\beta x_{i, 2}^{2}\right. \\
& +\lambda_{i, 1}\left[\max \left(a_{i}, x_{i-1,1}\right)+\frac{q_{1}}{u_{i, 1}}-x_{i, 1}\right] \\
& \left.+\lambda_{i, 2}\left[\max \left(x_{i, 1}, x_{i-1,2}\right)+\frac{q_{2}}{u_{i, 2}}-x_{i, 2}\right]\right\}
\end{aligned}
$$

Differentiating with respect to $u_{i, j}, x_{i, j}$, and $\lambda_{i, j}$ for $j=1,2$ we get the optimality equations:

$$
0=2 \alpha_{1} u_{i, 1}-\lambda_{i, 1} \frac{q_{1}}{u_{i, 1}^{2}}=2 \alpha_{2} u_{i, 2}-\lambda_{i, 2} \frac{q_{2}}{u_{i, 2}^{2}}
$$

the costate equations,

$$
\begin{aligned}
\lambda_{i, 1}= & \lambda_{i+1,1} \frac{d \max \left(a_{i+1}, x_{i, 1}\right)}{d x_{i, 1}} \\
& +\lambda_{i, 2} \frac{d \max \left(x_{i, 1}, x_{i-1,2}\right)}{d x_{i, 1}} \\
\lambda_{i, 2}= & 2 \beta x_{i, 2}+\lambda_{i+1,2} \frac{d \max \left(x_{i+1,1}, x_{i, 2}\right)}{d x_{i, 2}} \\
\lambda_{N, 1}= & 2 \beta x_{N, 2} \frac{\partial \max \left(x_{N-1,2}, x_{N, 1}\right)}{\partial x_{N, 1}} \\
\lambda_{N, 2}= & 2 \beta x_{N, 2}
\end{aligned}
$$

and the state equations (12),(13).

The costate equations clearly illustrate the difficulty with the tandem case. Critical jobs can occur either in the first server when $x_{i, 1}=a_{i+1}$ or in the second server when $x_{i, 1}=x_{i-1,2}$. While the costate equation for the second server is essentially identical to the costate equation for the single-server case, the costate equation for the first server is affected by the costates in the second server. This coupling is the source of the difficulty in trying to obtain an expression for the subdifferential. In the single-server case, when a job $i$ was critical, we could independently perturb its control to obtain the left and right derivatives of the augmented cost with respect to the control $u_{i}$. These left and right derivatives define the end points of the intervals whose union gives the subdifferential. In the tandem case, the coupling between the two servers prohibits such a simple result. Now the subdifferential is a collection of regions formed by taking left and right derivatives with respect to the controls for jobs in both servers. Only in simple cases is it possible to determine which jobs to perturb to obtain these regions.

To overcome this difficulty, we proceed as follows. Observe that in our hybrid system modeling framework, it 
is the presence of the max function in the costate equation that is the root of the problem described above. Except for the max function, all other functions involved are continuous and the problem could be easily solved using a standard gradient-based TPBVP solver. Therefore, we have proceeded by simply approximating the max function with a continuous surrogate. In particular, we "smooth" the max function by fitting it with a Bezier function (see Fig. 1). A Bezier function is constructed using $n+1$ "control points" represented by vectors $\mathbf{v}_{1}, \ldots, \mathbf{v}_{n}$ and is parametrically given by

$$
\mathbf{v}(t)=\sum_{i=0}^{n} \mathbf{v}_{i} B_{i, n}(t)
$$

where

$$
B_{i, n}(t)=\frac{n !}{i !(n-i) !} t^{i}(1-t)^{n-i}
$$

The control points define a "characteristic polygon" and the Bezier function has the property that it is contained within the convex hull of this characteristic polygon. In our case, there are three obvious control points to use: the point $(a, a)$ where the max function is not differentiable and two points $(a-\epsilon, a)$ and $(a+\epsilon, a+\epsilon)$ which define a neighborhood of $a$ on the $d$-axis in Fig. 1. An additional property of $\mathbf{v}(t)$ is that the tangents at the first and last control points coincide with the first and last segments of the characteristic polygon. In our case, the two properties imply that the derivative of the Bezier approximation of the max function is always between 0 and 1 . Using this approach with a standard gradient-based TPBVP solver, we periodically recompute the Bezier function and can make the fit arbitrarily tight by selecting the value of $\epsilon>0$. This value can be decreased and adjusted during the execution of the TPBVP solver to achieve any desired accuracy. In the limit, the solution obtained using this approach converges to the true optimal. The numerical results shown in Section 5 illustrate the effectiveness of this simple approach.

\section{Optimal Control Properties}

We begin by establishing some basic properties of the optimal control for the problem in (11). Our main result is that the optimal policy is one that requires the stage 2 control for all jobs to be fixed at a maximal rate after a number of initial jobs are processed.

Lemma 4.1 Let jobs $i$ and $i+1$ be in the same block of the jth stage. Then, the optimal controls satisfy

$$
u_{i, 1}^{*} \geq u_{i+1,1}^{*} \quad \text { and } \quad u_{i, 2}^{*}>u_{i+1,2}^{*}
$$

\section{$f(d)$}

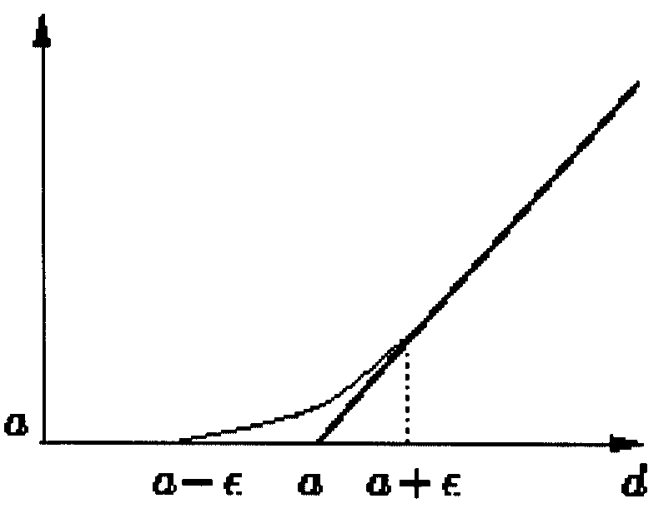

Figure 1: Bezier approximation of a max function.

Proof. Suppose jobs $i$ and $i+1$ are in the same block of the first stage, i.e., $a_{i+1}<x_{i, 1}$. Therefore,

$$
\begin{aligned}
\lambda_{i, 1}= & \lambda_{i+1,1} \frac{d \max \left(a_{i+1}, x_{i, 1}\right)}{d x_{i, 1}} \\
& +\lambda_{i, 2} \frac{d \max \left(x_{i, 1}, x_{i-1,2}\right)}{d x_{i, 1}} \\
= & \lambda_{i+1,1}+\lambda_{i, 2} \frac{d \max \left(x_{i, 1}, x_{i-1,2}\right)}{d x_{i, 1}}
\end{aligned}
$$

It follows from (15) that

$$
\begin{aligned}
u_{i, 1}^{*^{3}}-u_{i+1,1}^{*^{3}} & =\frac{\lambda_{i, 1} q_{1}}{2 \alpha_{1}}-\frac{\lambda_{i+1,1} q_{1}}{2 \alpha_{1}} \\
& =\frac{q_{1}}{2 \alpha_{1}}\left(\lambda_{i, 1}-\lambda_{i+1,1}\right) \\
& =\frac{q_{1}}{2 \alpha_{1}} \lambda_{i, 2} \frac{d \max \left(x_{i, 1}, x_{i-1,2}\right)}{d x_{i, 1}} \geq 0
\end{aligned}
$$

If jobs $i$ and $i+1$ are in the same block of the second stage, then $x_{i+1,1}<x_{i, 2}$. Therefore,

$$
\begin{aligned}
\lambda_{i, 2} & =2 \beta x_{i, 2}+\lambda_{i+1,2} \frac{d \max \left(x_{i+1,1}, x_{i, 2}\right)}{d x_{i, 2}} \\
& =2 \beta x_{i, 2}+\lambda_{i+1,2}
\end{aligned}
$$

It follows that

$$
\begin{aligned}
u_{i, 2}^{*^{3}}-u_{i+1,2}^{*^{3}} & =\frac{\lambda_{i, 2} q_{2}}{2 \alpha_{2}}-\frac{\lambda_{i+1,2} q_{2}}{2 \alpha_{1}} \\
& =\frac{q_{2}}{2 \alpha_{2}}\left(\lambda_{i, 2}-\lambda_{i+1,2}\right) \\
& =\frac{q_{2}}{\alpha_{2}} \beta x_{i, 2}>0
\end{aligned}
$$

which concludes the proof.

Lemma 4.2 The optimal controls $u_{i, 1}^{*}$ and $u_{i, 2}^{*}$ are bounded by $K_{1}<\infty$ and $K_{2}<\infty$ such that

$$
u_{i, 1}^{*} \leq K_{1}, \quad u_{i, 2}^{*} \leq K_{2} \quad \text { for all } i=1, \ldots, N
$$


Proof. Assume w.l.o.g that $u_{i, 1}^{*}=\infty$. Then, $J=\infty$. However, one can use, for example, $u_{i, 1}=u_{i, 2}=1$ which yields

$$
x_{j, 2} \leq a_{j}+q_{1}+q_{2}+(j-1) \max \left(q_{1}, q_{2}\right)
$$

that is, $x_{j, 2}$ is finite. Then,

$$
J=N\left(\alpha_{1}+\alpha_{2}\right)+\sum_{j=1}^{N} \beta x_{j, 2}^{2}<\infty
$$

for finite $N$ and the result follows.

Theorem 4.1 Assume that the optimal controls satisfy

$$
u_{j, 1}^{*} \leq K_{1}, \quad u_{j, 2}^{*} \leq K_{2} \quad \text { for all } j=1, \ldots, N
$$

for given $K_{1}<\infty$ and $K_{2}<\infty$. Then $u_{i, 2}^{*}=K_{2}$ for all $i \geq l$ where $l$ is the smallest integer satisfying

$$
\frac{\alpha_{2} K_{2}^{3} K_{1}}{\beta q_{1} q_{2}} \leq l
$$

Proof. Note that

$$
x_{l, 2}^{*} \geq \frac{q_{1}}{K_{1}} l+\frac{q_{2}}{K_{2}}>\frac{q_{1}}{K_{1}} l
$$

Assume that $i \geq l$ and the optimal control for the $i$ th job is $u_{i, 2}^{*}<K_{2}$. We will proceed by establishing a contradiction. Let us perturb the control $u_{i, 2}^{*}$ and evaluate the effect on the cost $J$. In particular, let $u_{i, 2}=u_{i, 2}^{*}+\delta_{i}$ for some $\delta_{i}>0$. First, note that

$$
\begin{aligned}
x_{i, 2} & =x_{i, 2}^{*}-\frac{q_{2}}{u_{i, 2}^{*}}+\frac{q_{2}}{u_{i, 2}} \\
& =x_{i, 2}^{*}-\frac{q_{2}}{u_{i, 2}^{*}}+\frac{q_{2}}{u_{i, 2}^{*}+\delta_{i}} \\
& =x_{i, 2}^{*}-\frac{q_{2} \delta_{i}}{u_{i, 2}^{*}\left(u_{i, 2}^{*}+\delta_{i}\right)}
\end{aligned}
$$

The change in cost is given by

$\Delta J=\alpha_{2}\left(u_{i, 2}^{2}-u_{i, 2}^{* 2}\right)+\beta\left(x_{i, 2}^{2}-x_{i, 2}^{2 *}\right)+\sum_{j=i+1}^{k} \beta\left(x_{j, 2}^{2}-x_{j, 2}^{2 *}\right)$

where $k$ denotes the last job of the busy period. Using (22) and the fact that $u_{i, 2}=u_{i, 2}^{*}+\delta_{i}$, we get

$$
\begin{aligned}
\Delta J= & \alpha_{2}\left(2 u_{i, 2}^{*}+\delta_{i}\right) \delta_{i}+\sum_{j=i+1}^{k} \beta\left(x_{j, 2}^{2}-x_{j, 2}^{2 *}\right) \\
& -\frac{\beta q_{2} \delta_{i}}{u_{i, 2}^{*}\left(u_{i, 2}^{*}+\delta_{i}\right)}\left(2 x_{i, 2}^{*}-\frac{q_{2} \delta_{i}}{u_{i, 2}^{*}\left(u_{i, 2}^{*}+\delta_{i}\right)}\right)
\end{aligned}
$$

The summation term above is always nonpositive and we have

$$
\begin{array}{r}
\Delta J \leq 2 \delta_{i}\left[\alpha_{2} u_{i, 2}^{*}-\frac{\beta q_{2}}{u_{i, 2}^{*}\left(u_{i, 2}^{*}+\delta_{i}\right)} x_{i, 2}^{*}\right] \\
+\delta_{i}^{2}\left[\alpha_{2}+\beta\left(\frac{q_{2}}{u_{i, 2}^{*}\left(u_{i, 2}^{*}+\delta_{i}\right)}\right)^{2}\right]
\end{array}
$$

Moreover, $x_{i, 2}^{*} \geq x_{l, 2}^{*}$ since $i \geq l$, therefore $x_{i, 2}^{*}>\frac{q_{1}}{K_{1}} l$ by (21). Using (20) and our assumption $u_{i, 2}^{*} \leq K_{2}$ implies that

$$
x_{i, 2}^{*}>\frac{\alpha_{2} u_{i, 2}^{* 3}}{\beta q_{2}}
$$

Therefore, for arbitrarily small (positive) $\delta_{i}$,

$$
\alpha_{2} u_{i, 2}^{*}-\frac{\beta q_{2}}{u_{i, 2}^{*}\left(u_{i, 2}^{*}+\delta_{i}\right)} x_{i, 2}^{*}<0
$$

and the remaining positive term in $\delta_{i}^{2}$ can be made small enough to ensure that $\Delta J<0$ which contradicts the assumption that the control sequence is optimal. Since increasing $u_{i, 2}$ decreases the cost $J$, the optimal control $u_{i, 2}^{*}=K_{2}$.

There are some interesting implications of the two lemmas and Theorem 4.1 above. If the controls at stage 2 are given by some maximal rate $K_{2}$ and $u_{i, 2}^{*}>u_{i+1,2}^{*}$ within a block by Lemma 4.1 , then every block can only contain a single job. This, in turn, implies that an optimal policy is eventually characterized by no buffering at stage 2 . However, formalizing this observation is still the subject of ongoing research, since the result of Theorem 1 a priori constrains the optimal controls to be bounded by given constants, whereas the two lemmas impose no such constraint.

\section{Numerical Results}

To illustrate our solution methodology, we consider a problem with $N=6$ jobs. These six jobs arrive at given times $0.2,1.3,1.4,1.8$, and 2.5 seconds, respectively. Each job is processed in the first server until it reaches a physical state $q_{1}=0.5$, and in the second server until it reaches a physical state $q_{2}=0.6$. The cost function (11) uses parameters $\alpha_{1}=2, \alpha_{2}=3$, and $\beta=2$. Figure 2 shows the evolution of the control for each job in each server (plots labeled ' $u 1$ ' and ' $u 2$ '), and the evolution of the total cost (plot labeled 'Cost $J^{\prime}$ ). The final sample path (queue length vs. time) for each server is shown in Figure 3. Note that the cost and controls quickly converge and the presence of critical jobs at stage 2 (as seen in Figure 3) is still captured by the Bezier approximation. Moreover, the absence of buffering observed at stage 2 is consistent with the discussion of the previous section. 


\section{References}

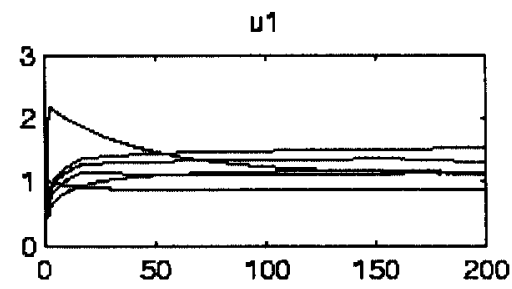

42

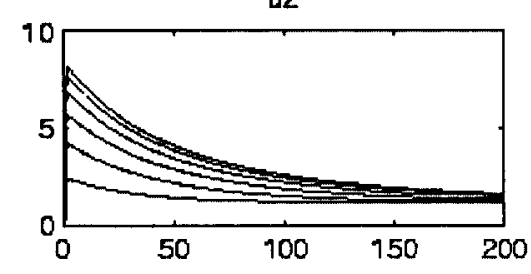

Cost J

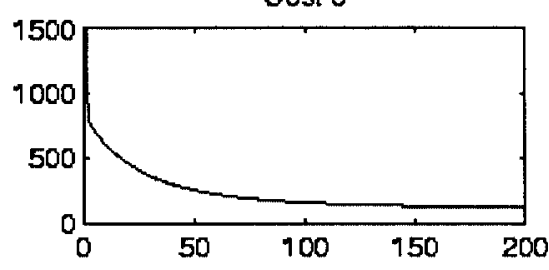

Figure 2:
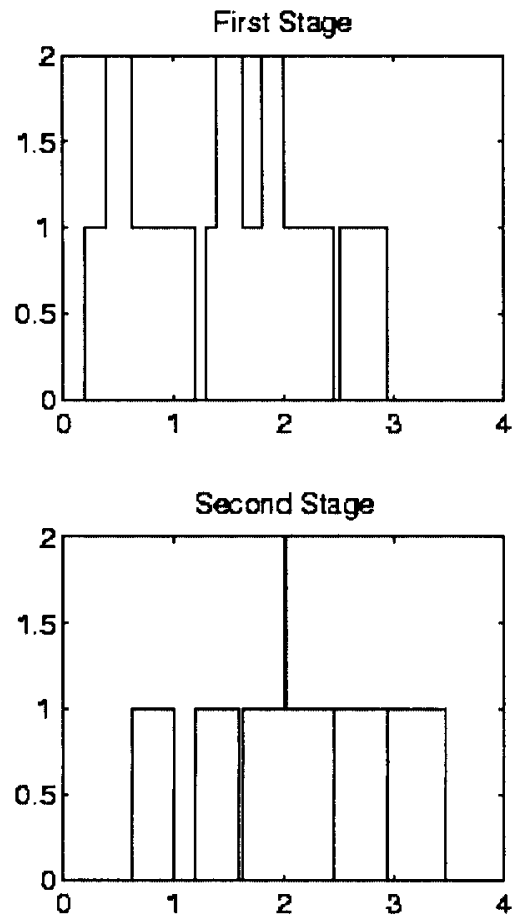

Figure 3:
[1] Alur, A., T. A. Henzinger, and E. D. Sontag, eds., Hybrid Systems. Springer-Verlag, 1996.

[2] Antsaklis, P., W. Kohn, M. Lemmon, A. Nerode, and S. Sastry, eds., Hybrid Systems. SpringerVerlag, 1998.

[3] Branicky, M. S., V. S. Borkar, and S. K. Mitter, "A unified framework for hybrid control: Model and optimal control theory," IEEE Tr. on Automatic Control, vol. 43, no. 1, pp. 31-45, 1998.

[4] Cassandras, C. G. Discrete Event Systems: Modeling and Performance Analysis. Irwin Publ., 1993.

[5] Cassandras, C. G., and D. L. Pepyne, "Optimal control of a class of hybrid systems," Proceedings of the 36th IEEE Conference on Decision and Control, 1997.

[6] Cassandras, C. G., D. L. Pepyne, and Y. Wardi, "Generalized gradient algorithms for hybrid system models of manufacturing systems," in Proc. Of 37th IEEE Conf. On Decision and Control, pp. 2627-2632, December 1998.

[7] Cassandras, C. G., D. L. Pepyne, and Y. Wardi, "Optimal control of systems with time-driven and event-driven dynamics," in Proc. Of 37 th IEEE Conf. On Decision and Control, pp. 7-12, December 1998 .

[8] Cassandras, C. G., D. L. Pepyne, and Y. Wardi, "Optimal control of hybrid system models of manufacturing systems," 1999.

[9] Cassandras, C. G., D. Pepyne, and Y. Wardi, "Optimal control of hybrid systems and some manufacturing applications," Proceedings of the 1998 Workshop on Discrete Event Systems, 1998.

[10] Clarke, F. H. Optimization and Nonsmooth Analysis. Wiley-Interscience, 1983.

[11] Grossman, R. L., A. Nerode, A. P. Ravn, and H. Rischel, eds., Hybrid Systems - Vol. 736 of Lecture Notes in Computer Science. Springer-Verlag, 1993.

[12] Pepyne, D. L, and C. G. Cassandras, "Modeling, analysis, and optimal control of a class of hybrid systems," Journal of Discrete Event Dynamic Systems: Theory and Applications, vol. 8, no. 2, pp. 175-201, 1998. 